\title{
Der geriatrische Patient im Mittelpunkt - AO-Kurs Alterstraumatologie in Erfurt
}

\author{
Stephanie Schibur, Thomas Mückley, Edgar Mayr
}

Mit namhaften chirurgischen, aber auch ganz bewusst nichtchirurgischen Experten als Referenten startete Ende Mai 2019 der AO-Kurs Alterstraumatologie in Erfurt unter der Leitung von Professor Mückley und Professor Mayr. Ziel war es, den betagten Patienten in seiner Gesamtheit in den Mittelpunkt der Diskussion zu rücken und die Kursteilnehmer mit einem Arbeitszeug auszustatten, das sie befähigt, die komplexen Fragestellungen, die sich bei der Versorgung dieser Patientengruppe ergeben, zu bewältigen.

Konsequenterweise startete der 1. Kurstag mit dem präoperativen Management geriatrischer Patienten. Wie ein Mantra zog sich der Grundsatz, dass der Sturz des alten Menschen bis zum Beweis des Gegenteils als Dekompensation einer (vorbestehenden) Grunderkrankung gesehen werden muss, durch die einzelnen Vorträge. Klar ersichtlich wurde durch diese Vorgehensweise, dass ein Therapiekonzept, welches nur die Verletzungsfolgen detektiert und behandelt, den Patienten nicht kausal versorgt. Unverzichtbar für die Behandlung von geriatrischen Patienten ist ein antikoagulatives Management mit Augenmaß. Um dieses wenig chirurgische Thema für den Arbeitsalltag in Notaufnahmen und Ambulanzen verständlich und sicher umsetzbar zu vermitteln, schloss sich ein Übersichtsvortrag und eine Handlungsempfehlung für den Umgang mit NOAKs an.
Dauerbrenner in der alterstraumatologischen Versorgung sind die periprothetischen und periimplantären Frakturen. Diesem Thema wurde eine eigene Sitzung gewidmet. An zwei umfassende Übersichtsvorträge schloss sich ein bewertender Vortrag mit dem Ziel an, evidenzbasiertes Wissen in den Alltag zu übertragen. Die rege Diskussion war nicht zuletzt von den Erfahrungen der Kursteilnehmer gestärkt. Ebenso wurde dem Thema Sprunggelenkfrakturen im Alter ein eigener Themenblock gewidmet. Innovative Implantate, Standards der Behandlung, die Gefäßabklärung und Intervention sowie die mögliche Deckung von Weichteildefekten wurden ausführlich behandelt.

Geriatrische Becken- und Wirbelkörperfrakturen sind eine eigene Entität. Bewährte Klassifikationen und Therapien lassen sich nicht immer auf osteoporotische Frakturen übertragen. Auch hier kann man einen rasanten Erkenntnisgewinn verzeichnen. Um eine breite Basis für eine angeregte Diskussion zu schaffen, wurden die Referenten in diesen Sitzungen zum „Science Slam“ - einem wissenschaftlichen Schlagabtausch - geladen. Kontroverse Ansichten waren gewünscht und ließen Raum dafür, Fallstricke zu erörtern und von Stolpersteinen berichtet zu bekommen.

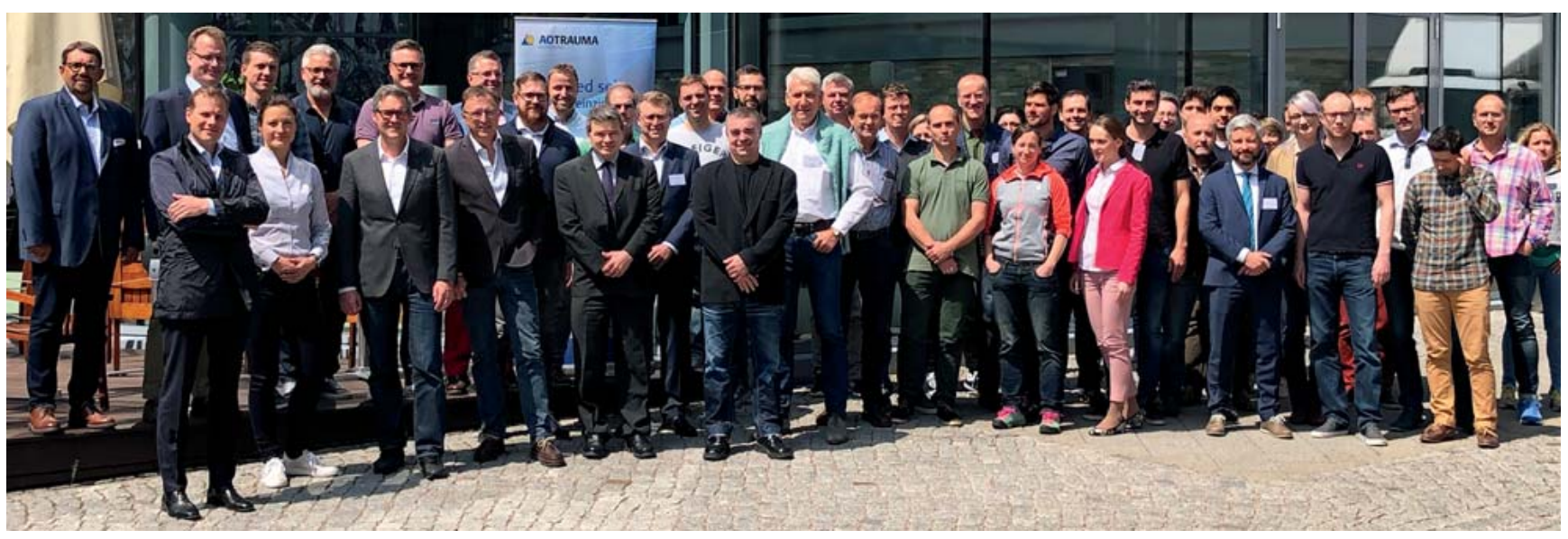

Abb. 1 Teilnehmer und Referenten des Kurses. Quelle: Edgar Rieger 


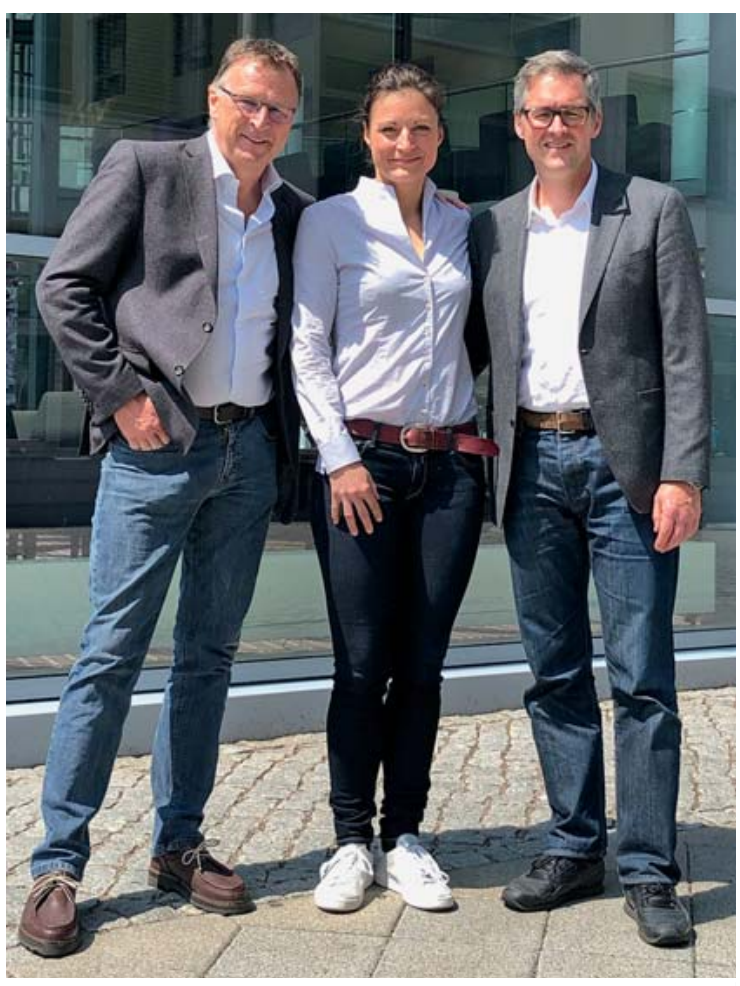

- Abb. 2 Die Kursleiter Mayr und Mückley mit OA Dr. Schibur. Quelle: Edgar Rieger

Neben den Frakturen am proximalen und distalen Femur wurden auch die proximalen Tibiafrakturen im Alter in Einzelreferaten umfassend dargestellt. Die Behandlungsprinzipien haben sich in den letzten Jahren in der Alterstraumatologie weiterentwickelt, unter anderem mit dem einheitlichen Ziel, eine frühe Vollbelastbarkeit herzustellen.
Die Osteoporosediagnostik und -therapie durften selbstverständlich nicht fehlen. Ebenso das Komplikationsmanagement von infizierten Prothesen und infizierten Osteosynthesen in der Alterstraumatologie. Andrej Trampuz von der Charité Berlin konnte diesbezüglich alle Kursteilnehmer von der zunehmenden Standardisierung und Etablierung von Behandlungsalgorithmen mit einem herausragenden Vortrag überzeugen.

Abgerundet wurde das Programm dank Benjamin Bücking, der als Leiter der AG Alterstraumatologie den Kursteilnehmern hilfreiche Tipps für einen erfolgreichen Zertifizierungsprozess zum ATZ DGU mit auf den Weg gab.

Unser herzlicher Dank gilt den ambitionierten Referenten und diskussionsfreudigen Kursteilnehmern, die diese Veranstaltung so interaktiv genutzt haben, wie wir sie uns wünschten - immer mit dem Ziel, die Versorgung der alten verunfallten Patienten flächendeckend zu optimieren.

Stephanie Schibur, Erfurt Thomas Mückley, Erfurt Edgar Mayr, Augsburg

Bibliografie

DOI https://doi.org/10.1055/a-0953-5419

OP-JOURNAL 2019; 35: 202-203 @ Georg Thieme Verlag KC Stuttgart · New York ISSN 0178-1715 